\title{
Vasculoprotective Effects of Combined Endothelial Progenitor Cells and Mesenchymal Stem Cells in Diabetic Wound Care: Their Potential Role in Decreasing Wound-Oxidative Stress
}

\author{
Supakanda Sukpat, ${ }^{1}$ Nipan Isarasena, ${ }^{2}$ Jutamas Wongphoom, ${ }^{3}$ and Suthiluk Patumraj ${ }^{4}$ \\ ${ }^{1}$ Inter-Department of Physiology, Graduate School, Faculty of Medicine, Chulalongkorn University, Bangkok 10330, Thailand \\ ${ }^{2}$ Stem Cell and Cell Therapy Research Unit, Department of Pharmacology, Faculty of Medicine, Chulalongkorn University, \\ Bangkok 10330, Thailand \\ ${ }^{3}$ King Chulalongkorn Memorial Hospital, Bangkok 10330, Thailand \\ ${ }^{4}$ Department of Physiology, Center of Excellence for Microcirculation, Faculty of Medicine, Chulalongkorn University, \\ Bangkok 10330, Thailand \\ Correspondence should be addressed to Suthiluk Patumraj; suthilukp@yahoo.com
}

Received 9 March 2013; Revised 10 May 2013; Accepted 15 May 2013

Academic Editor: Dimitrios P. Bogdanos

Copyright @ 2013 Supakanda Sukpat et al. This is an open access article distributed under the Creative Commons Attribution License, which permits unrestricted use, distribution, and reproduction in any medium, provided the original work is properly cited.

\begin{abstract}
To investigate whether the combined endothelial progenitor cells (EPCs) and mesenchymal stem cells (MSCs) could enhance angiogenesis and wound healing in diabetic mice. Balb/c nude mice were divided into five groups, including a control group, diabetic group $(\mathrm{DM})$, DM injected with $1 \times 10^{6}$ cells MSCs, DM injected with $1 \times 10^{6}$ cells EPCs, and DM injected with combined $0.5 \times 10^{6}$ cells MSCs and $0.5 \times 10^{6}$ cells EPCs. After seven weeks, the mice were anesthetized, and bilateral full-thickness excision skin wounds were made on the dorsorostral back. The percentage of wound closure in DM group decreased significantly than in control and all other treated groups on day 7 and day $14(P<0.005)$. On day 14 , the percentage of capillary vascularity in combinetreated group was significantly higher than in $\mathrm{DM}(P<0.005)$. In the present study, we have demonstrated that the combined EPCs and MSCs can increase vascular endothelial growth factor (VEGF) level and angiogenesis which resulted in reduced neutrophil infiltration, decreased malondialdehyde (MDA) levels, and enhanced wound healing in diabetic mice model.
\end{abstract}

\section{Introduction}

A diabetic wound is characterized by poor circulation and impaired angiogenesis, which contributes to nonhealing ulcers and may result in subsequent amputation. In addition, $84 \%$ of amputations among diabetic foot-ulcer patients have been reported [1-3].

Diabetes-induced endothelium and vascular dysfunction are closely associated with oxidative stress due to hyperglycemia caused by the excessive generation of reactive oxygen species (ROS). It has also been suggested that ROS can reduce the number and function of EPCs [4]. Consequently, EPC dysfunction may decrease the vascular regenerative potential of diabetic patients and thereby contribute to the pathogenesis of diabetic ulcers [4]. Moreover, high levels of superoxides in the diabetic wound may result in abnormalities in the number of local cytokines in the wound as well as reduce the production of growth factors, such as plateletderived growth factor (PDGF), transforming growth factor$\beta$ (TGF- $\beta$ ), and vascular endothelial growth factor (VEGF), which contribute to the delay in wound healing [5].

Recently, several studies have suggested that MSCs can promote wound healing, angiogenesis, and the release of proangiogenic factors, including VEGF, basic fibroblast growth factor (bFGF) and stromal-derived factor-1 (SDF1) [6-8]. Thus, this study aimed to examine the effect of combined EPCs and MSCs treatment on angiogenesis and wound healing in diabetic mice. In particular, we assessed the 
levels of ROS in the wound area where the EPCs and MSCs were injected. We hypothesized that the vasculoprotective effect of these combined EPCs and MSCs may be due to their ability to decrease ROS levels in the diabetic wound area.

\section{Materials and Methods}

2.1. Animal Preparation. Male Balb/c nude mice (7-8 weeks old, 20-25 g) were purchased from the National Laboratory Animal Center, Salaya Campus, Bangkok, Thailand. The experimental procedures were conducted according to the guideline of experimental animals by The National Research Council of Thailand and were approved by the ethics committee at the Faculty of Medicine, Chulalongkorn University. The mice were housed at room temperature $\left(25 \pm 3^{\circ} \mathrm{C}\right)$ with one mouse per cage and fed with standard chow and sterilized water. The mice were divided into five groups.

Group 1: wound control group with implanted fibrin gel (control).

Group 2: diabetic wound group with implanted fibrin gel (DM).

Group 3: diabetic wound group with implanted fibrin gel and $1 \times 10^{6}$ MSCs (DM + MSCs).

Group 4: diabetic wound group with implanted fibrin gel and $1 \times 10^{6}$ EPCs (DM + EPCs).

Group 5: diabetic wound group with implanted fibrin gel and combined $0.5 \times 10^{6} \mathrm{EPCs}$ and $0.5 \times 10^{6} \mathrm{MSCs}$ (DM + EPCs + MSCs).

Animals in the diabetic groups were induced by an injection of streptozotocin (STZ, Sigma Chemical Co., USA) in citrate buffer, $\mathrm{pH} 4.5$ (Sigma Chemical Co., USA) at a dose of $45 \mathrm{mg} / \mathrm{kg}$ i.p. daily for 5 days. Two weeks later, the glucose level was measured from tail-vein blood, and mice with glycemia (over $200 \mathrm{mg} / \mathrm{dL}$ ) were selected for the study [9].

2.2. Preparation of Mouse MSCs. Mouse MSCs were cultured in Dulbecco's Modified Eagle's Medium (DMEM) containing $5 \%$ fetal bovine serum (FBS), 1x Pen-strep, and L-glutamine at $37^{\circ} \mathrm{C}, 5 \% \mathrm{CO}_{2}$. The medium was changed every 2 or 3 days. The cells were maintained by the Stem Cell and Cell Therapy Laboratory.

2.3. Preparation of Human EPCs. Human EPCs were purchased from Lonza. The cells were plated on culture dishes coated with fibronectin (Gibco) and cultured in endothelial cell basal medium 2 (EBM-2, Lonza) at $37^{\circ} \mathrm{C}$ and $5 \% \mathrm{CO}_{2}$. The medium was changed every 2 or 3 days. The cells were maintained by the Stem Cell and Cell Therapy Laboratory.

2.4. Preparation of Stem Cells and Fibrin Gel. The cells were washed with sterile phosphate-buffered saline (PBS) and then incubated with $2 \mathrm{~mL} 0.25 \%$ trypsin for 1 minute at $37^{\circ} \mathrm{C}$. Next, $10 \mathrm{~mL}$ of culture medium was added to the plate to neutralize the trypsin activity. The cell suspension was transferred into a tube, and the number of cells was quantified using a microscope at 100x magnification. The cells were then centrifuged for $5 \mathrm{~min}$ at $1000 \mathrm{rpm}$ at room temperature. The supernatant was removed, and the cell pellet was mixed with $30 \mu \mathrm{L}$ fibrinogen and $30 \mu \mathrm{L}$ thrombin per wound (the fibrin gel consisted of fibrinogen and thrombin and was obtained from Shanghai RAAS Blood Products Co. Ltd, China) [10].

2.5. Wound Model. Six to seven weeks after the streptozotocin injection, the mice were anesthetized by an intraperitoneal injection of sodium pentobarbital at a dose of $55 \mathrm{mg} / \mathrm{kg}$ and an alcohol swab was used on its dorsorostral back. Bilateral full-thickness excision skin wounds $\left(0.6 \times 0.6 \mathrm{~cm}^{2}\right)$ were created on each side of the midline [8]. Each mouse received fibrin gel (control and DM), fibrin gel and $1 \times 10^{6}$ MSCs (DM + MSCs), fibrin gel and $1 \times 10^{6}$ EPCs (DM + EPCs), and fibrin gel with combined $0.5 \times 10^{6}$ MSCs and 0.5 $\times 10^{6}$ EPCs $(\mathrm{DM}+\mathrm{EPCs}+\mathrm{MSCs})$ on the wound bed. The wounds were then covered using Tegaderm (3M, USA). The EPCs and MSCs were maintained by the Stem Cell and Cell Therapy Research Unit at Chulalongkorn University. In this study, we would like to test the effect of human EPCs on diabetic wound (DM + EPCs group) and to compare with another reference that used only mouse MSCs [8], and also with the combined group. Therefore, we need to develop the diabetic model in nude mice, which demonstrate an immune deficiency.

2.6. Wound Analysis. Digital images of the wounds were taken at days 0,7 and 14. The wound area was analyzed using digital image software analysis (Image Pro II 6.1). The percentage of wound closure (\%WC) was calculated using the following equation: (area of the original wound - area of the actual wound)/area of the original wound $\times 100$ [8].

2.7. Determination of Capillary Vascularity. At days 7 and 14 following wounding, the mice were anesthetized with an intraperitoneal (IP) injection of sodium pentobarbital at a dose of $55 \mathrm{mg} / \mathrm{kg}$. The jugular vein was cannulated for injection of $0.2 \mathrm{~mL}$ of $5 \%$ FITC-labeled dextran (MW $=250000$, Sigma Chemical Co., USA). The percentage of capillary vascularity $(\mathrm{CV})$ was examined using intravital fluorescence video microscopy with a 10x objective lens [11]. From the video images, the percentage of capillary vascularity (\%CV) was analyzed using Image Pro II 6.1 software. The region of interest $(\mathrm{ROI})$ was $100 \times 100$ pixels. Each ROI was selected to cover only capillary networks with a diameter of less than $15 \mu \mathrm{m}[11,12]$. The average CV consisted of at least three ROIs from each animal.

These results were confirmed by a blind assessment. The $\% \mathrm{CV}$ was calculated using the following formula: (number of pixels within the capillaries/total number of pixels within the select area) $\times 100[11,12]$.

2.8. Measurement of Neutrophil Infiltration. The mice were sacrificed at 7 and 14 days, and the wound samples were harvested at a size of $0.6 \times 0.6 \mathrm{~cm}^{2}$. The tissue specimens were fixed in $10 \%$ formaldehyde for 24 hours. The center of 
TABLE 1: The means \pm SEM of the body weight and blood glucose on day 7 and 14 after wounding in the wound control group with implanted fibrin gel (control), diabetic wound group with implanted fibrin gel (DM), diabetic wound group with implanted fibrin gel and $1 \times 10^{6}$ MSCs $(\mathrm{DM}+\mathrm{MSCs})$, diabetic wound group with implanted fibrin gel and $1 \times 10^{6} \mathrm{EPCs}(\mathrm{DM}+\mathrm{EPCs})$, and diabetic wound group with implanted fibrin gel and combined $0.5 \times 10^{6}$ EPCs and $0.5 \times 10^{6}$ MSCs (DM + EPCs + MSCs).

\begin{tabular}{lcccc}
\hline \multirow{2}{*}{ Group } & \multicolumn{2}{c}{ Body weight $(\mathrm{g})$} & \multicolumn{2}{c}{ Blood glucose level (mg/dL) } \\
& Day 7 & Day 14 & Day 7 & Day 14 \\
\hline Control & $25.91 \pm 1.22$ & $26.06 \pm 1.45$ & $115.17 \pm 14.28$ & $120.40 \pm 11.52$ \\
DM & $23.86 \pm 2.07$ & $24.77 \pm 0.72$ & $347.83 \pm 59.41^{* *}$ & $362.83 \pm 57.56^{* *}$ \\
DM + MSCs & $21.81 \pm 1.40$ & $22.61 \pm 2.32$ & $361.33 \pm 18.63^{* *}$ & $425.00 \pm 104.74^{* *}$ \\
DM + EPCs & $24.76 \pm 1.41$ & $22.86 \pm 1.30$ & $344.17 \pm 61.21^{* *}$ & $362.40 \pm 35.56^{*}$ \\
DM + EPCs + MSCs & $24.57 \pm 1.07$ & $23.60 \pm 0.71$ & $312.33 \pm 15.05^{* *}$ & $376.50 \pm 82.19^{*}$ \\
\hline
\end{tabular}

${ }^{*} P<0.05$ : significant difference compared to control group.

${ }^{* *} P<0.01$ : significant difference compared to control group.

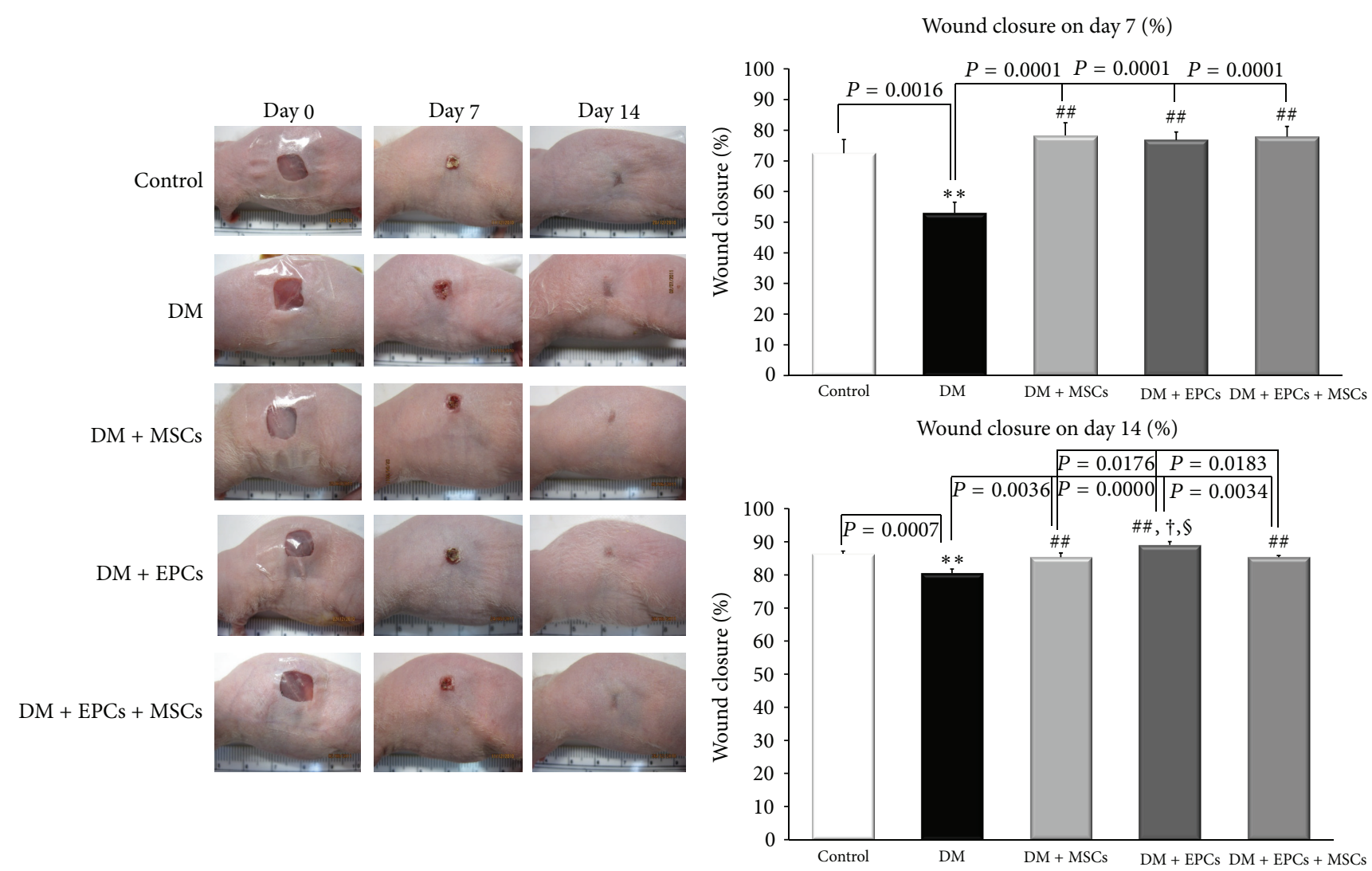

FIGURE 1: The percentages of wound closure in the control group with implanted fibrin gel (control), diabetic wound group with implanted fibrin gel (DM), diabetic wound group with implanted fibrin gel and MSCs (DM + MSCs), diabetic wound group with implanted fibrin gel and EPCs (DM + EPCs), and diabetic wound group with implanted fibrin gel and combined MSCs and EPCs (DM + MSCs + EPCs) on day 7 and day 14 are shown. The data are presented as the means \pm SEM. ${ }^{* *} P<0.005$ : significant difference compared to the control group. ${ }^{\#} P<0.005$ : significant difference compared to the DM group. ${ }^{\dagger} P<0.05$ : significant difference compared to the DM + MSCs + EPCs group. ${ }^{\S} P<0.05$ : significant difference compared to the DM + MSCs group.

the wound samples was cut and embedded in paraffin. Fourmicrometer thick sections were stained with hematoxylineosin (H\&E). Neutrophil infiltration was measured using a microscope (Nikon E50i, Japan) at 400x magnification. The inflammatory wound area of the specimens was evaluated. The wound image was captured by a digital camera (Nikon DS-L2, Japan), and the amount of neutrophil infiltration was analyzed using Image Pro II 6.1 software [12]. These results were also confirmed by blind assessment.

2.9. Determination of Tissue VEGF, Malondialdehyde Levels, and Total Tissue Protein. The tissue sample was harvested from each mouse at 7 and 14 days following wounding and 

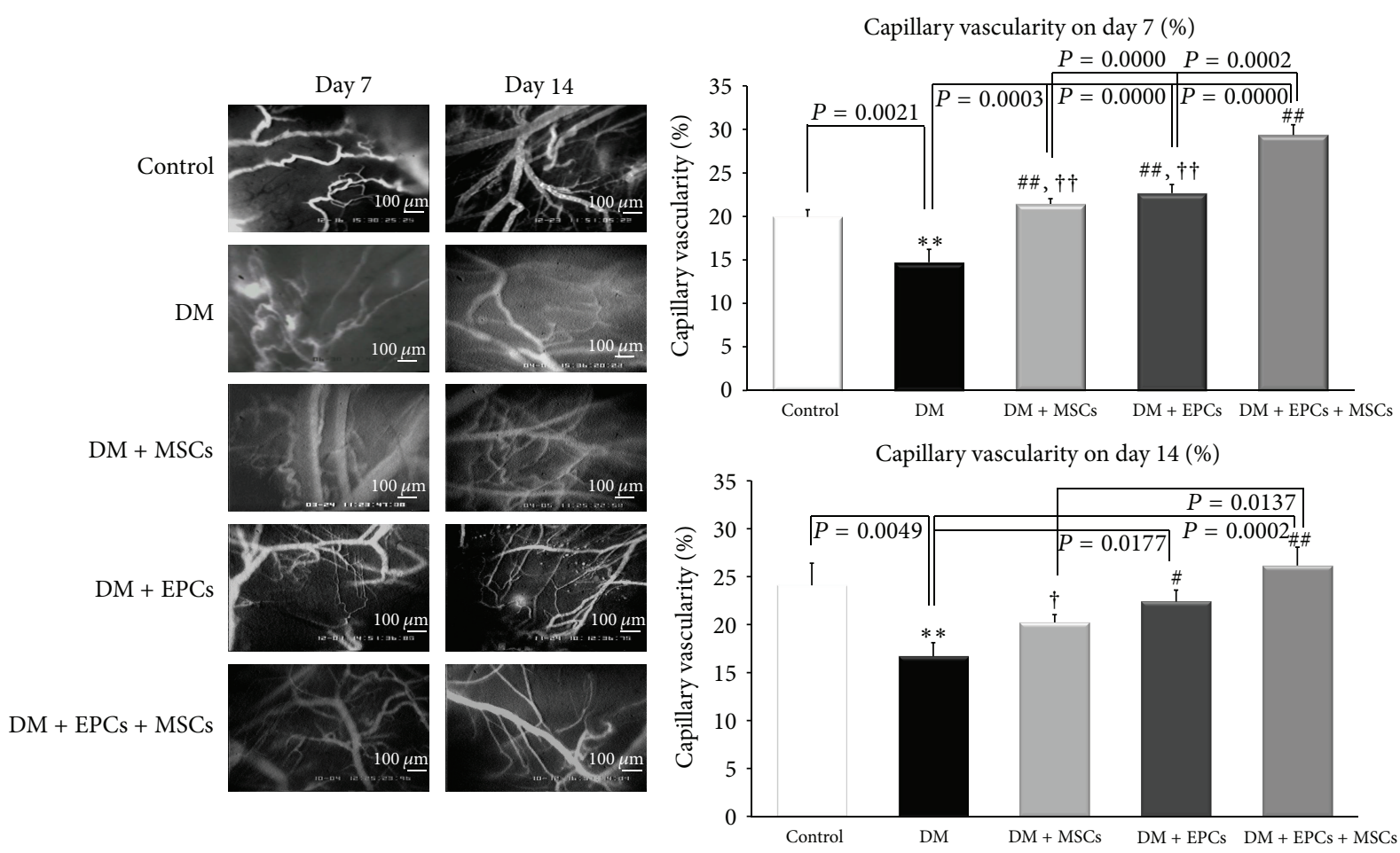

FIGURE 2: Percentages of capillary vascularity in the control group with implanted fibrin gel (control), diabetic wound group with implanted fibrin gel (DM), diabetic wound group with implanted fibrin gel and MSCs (DM + MSCs), diabetic wound group with implanted fibrin gel and EPCs (DM + EPCs), and diabetic wound group with implanted fibrin gel and combined MSCs and EPCs (DM + MSCs + EPCs) on day 7 and day 14 are shown. The data are presented as the means \pm SEM. ${ }^{* *} P<0.005$ : significant difference compared to the control group. ${ }^{\#} P<0.05$ : significant difference compared to the DM group. ${ }^{\#} P<0.005$ : significant difference compared to the DM group. ${ }^{\dagger} P<0.05$ : significant difference compared to the DM + MSCs + EPCs group. ${ }^{\dagger \dagger} P<0.001$ : significant difference compared to the DM + MSCs + EPCs group.

frozen at $-80^{\circ} \mathrm{C}$. A $50 \mathrm{mg}$ tissue sample was homogenized in $50 \mathrm{~mL}$ RIPA lysis buffer (Cell Signaling Technology, Inc.) with protease inhibitor (Sigma Chemical Co., USA) and phosphatase inhibitor cocktails (Sigma Chemical Co., USA). The sample was sonicated and centrifuged at 10,000 rpm for $10 \mathrm{~min}$. The supernatants of each tissue sample were used to analyze the levels of VEGF, malondialdehyde (MDA; lipid peroxides parameter), and the total tissue protein using ELISA (R\&D Systems), TBARS assay kit (Cayman Chemical Co., USA) and microplate BCA protein assay kit (Thermo Scientific, USA), respectively. The VEGF and MDA levels were expressed as $\mathrm{pg} / \mathrm{mg}$ protein and $\mathrm{nM} / \mathrm{mg}$ protein, respectively.

2.10. Statistical Analysis. All of the data were presented as the mean \pm SEM, and comparison between groups was conducted using one-way ANOVA, followed by the Least Significant Difference posttest (LSD). The correlation analysis was conducted using the two-tailed Pearson's correlation. Differences were considered statistically significant at a $P$ value less than $0.05(P<0.05)$.

\section{Results and Discussion}

The results of body weight and blood glucose are shown for each group (Table 1). On days 7 and 14, the blood sugar in the DM, DM + MSCs, DM + EPCs, and DM + EPCs + MSCs groups were significantly higher than their age-matched controls. The body weights of the all four groups of diabetes tend to decrease; however, they were not shown to be of statistical significance; that may be due to the small number of each group and high variability (Table 1).

3.1. Effects of EPCs and MSCs on Wound Closure. On day 7 and day 14 of postoperative wound, the percentage of wound closure (WC) in all of the treatment groups and control group was significantly increased compared to the DM group $(P<$ 0.005; Figure 1). Interestingly, 14 days after wounding, the $\% \mathrm{WC}$ of the DM + EPCs group was significantly increased compared to the DM + MSCs and DM + EPCs + MSCs groups $(P<0.05$; Figure 1$)$.

3.2. Effects of EPCs and MSCs on Angiogenesis. The results on day 7 showed that the $\% \mathrm{CV}$ in all of the treatment groups and control group was significantly increased compared to the DM group $(P<0.005$; Figure 2$)$. Moreover, these results indicated that, on day 7 and day 14 , the $\% \mathrm{CV}$ of the DM + EPCs + MSCs group was significantly higher compared to the values of the DM and DM + MSCs groups $(P<0.001$, $P<0.05$; Figure 2). 


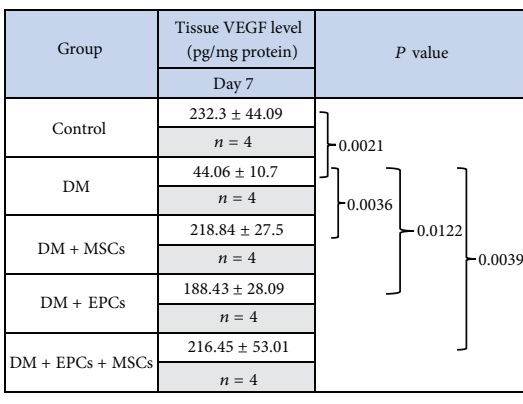

\begin{tabular}{|c|c|c|}
\hline \multirow[t]{2}{*}{ Group } & $\begin{array}{c}\text { Tissue VEGF level } \\
\text { (pg/mg protein) }\end{array}$ & \multirow[t]{2}{*}{$P$ value } \\
\hline & Day 14 & \\
\hline \multirow{2}{*}{ Control } & $45.3 \pm 7.91$ & \\
\hline & $n=4$ & \\
\hline \multirow{2}{*}{ DM } & $28.18 \pm 2.19$ & \\
\hline & $n=4$ & \\
\hline \multirow{2}{*}{$\mathrm{DM}+\mathrm{MSCs}$} & $44.99 \pm 9.58$ & \\
\hline & $n=4$ & \\
\hline \multirow{2}{*}{$\mathrm{DM}+\mathrm{EPCs}$} & $40.36 \pm 12.86$ & \\
\hline & $n=5$ & \\
\hline \multirow{2}{*}{$\mathrm{DM}+\mathrm{EPCs}+\mathrm{MSCs}$} & $50.73 \pm 8.97$ & \\
\hline & $n=4$ & \\
\hline
\end{tabular}

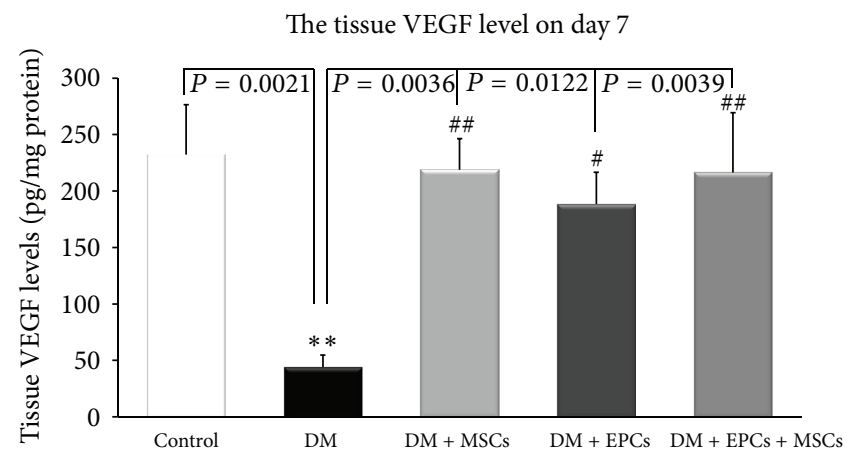

The tissue VEGF level on day 14

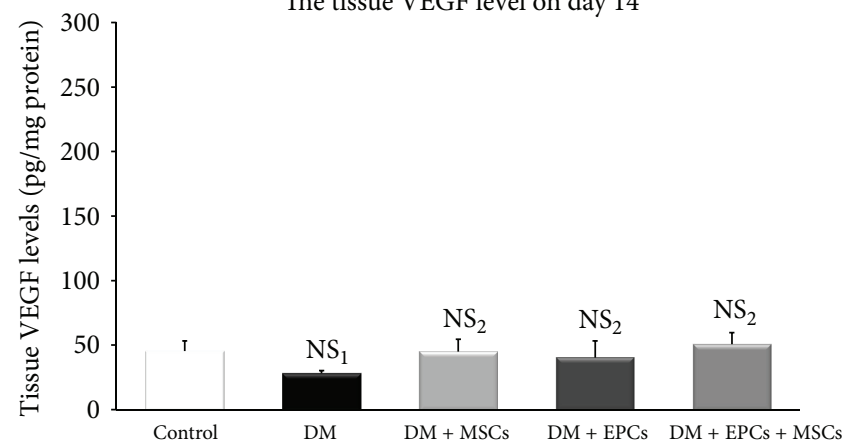

FIGURE 3: Effects of EPCs and MSCs on tissue VEGF levels. The tissue VEGF levels in the control group with implanted fibrin gel (control), diabetic wound group with implanted fibrin gel (DM), diabetic wound group with implanted fibrin gel and MSCs (DM + MSCs), diabetic wound group with implanted fibrin gel and EPCs (DM + EPCs), and diabetic wound group with implanted fibrin gel and combined MSCs and EPCs (DM + MSCs + EPCs) on day 7 and day 14 are shown. The data are presented as the means \pm SEM. ${ }^{* *} P<0.005$ : significant difference compared to the control group. ${ }^{\#} P<0.05$ : significant difference compared to the DM group. ${ }^{\# \#} P<0.005$ : significant difference compared to the DM group. $\mathrm{NS}_{1}$ : no significant difference compared to the control group. $\mathrm{NS}_{2}$ : no significant difference compared to the DM group.

3.3. Effects of EPCs and MSCs on Tissue VEGF Levels. The tissue VEGF levels of all of the treatment groups and control group were significantly increased compared to the DM group only on day 7 (Figure 3). There was no significant difference in the tissue VEGF levels among the groups on day 14 .

\subsection{Effects of Combined EPCs and MSCs on Neutrophil} Infiltration. The number of neutrophils infiltrating into the wound area between the five groups is shown in Figure 4. On day 7, the number of infiltrating neutrophils in the DM group significantly increased compared to the control group $(P=0.02)$. Moreover, the DM + MSCs, DM + EPCs, and $\mathrm{DM}+\mathrm{EPCs}+\mathrm{MSC}$ groups showed no significant difference when compared to the DM group (Figure 4).

On day 14, the number of infiltrating neutrophils in the DM group significantly increased compared to the control group $(P=0.011)$. The DM + MSCs, DM + EPCs, and DM + EPCs + MSCs groups showed a significantly reduced number of neutrophils compared to the DM group $(P=0.004, P=$ $0.006, P=0.015$, resp.); however, there was no significant difference among the DM + MSCs, DM + EPCs, and DM + EPCs + MSCs groups (Figure 4).

3.5. Effects of Combined EPCs and MSCs Treatment on Tissue MDA Levels. On day 7 and day 14 of postoperative wound, the tissue MDA levels in the DM group were significantly higher compared to all of the other groups (Figure 5). Interestingly, at 7 days after wounding, the tissue MDA levels in the DM + EPCs + MSCs group were significantly decreased compared to the DM and DM + MSCs groups $(P<0.01, P<$ 0.05; Figure 5). However, there was no significant difference between the DM + EPCs + MSCs and DM + EPCs groups.

In the present study, to induce type 1 diabetes mellitus in nude mice, multiple low doses of STZ $(45 \mathrm{mg} / \mathrm{kg}$ body weight) were administered to the mice daily for 5 days. STZ chemically damages pancreatic $\beta$ cells by DNA damage and results in hypoinsulinemia and hyperglycemia $[13,14]$. As shown in the present study, the blood glucose levels in the DM, DM + MSCs, DM + EPCs, and DM + EPCs + MSCs groups were significantly higher than those in the controls for both of the studied periods (Table 1). These results also showed no effect of EPCs and MSCs on the changes in blood glucose levels.

Diabetes is characterized by the reduced production of growth factors, poor circulation, and impaired angiogenesis, which contribute to poor wound healing, which has been reported in diabetic patients. VEGF, an important growth factor that induces angiogenesis, was also reduced in the DM group [15]. The present results showed that on day 7, tissue VEGF levels were significantly increased in the DM + MSCs, $\mathrm{DM}+\mathrm{EPCs}$ and DM + EPCs + MSCs groups compared to 

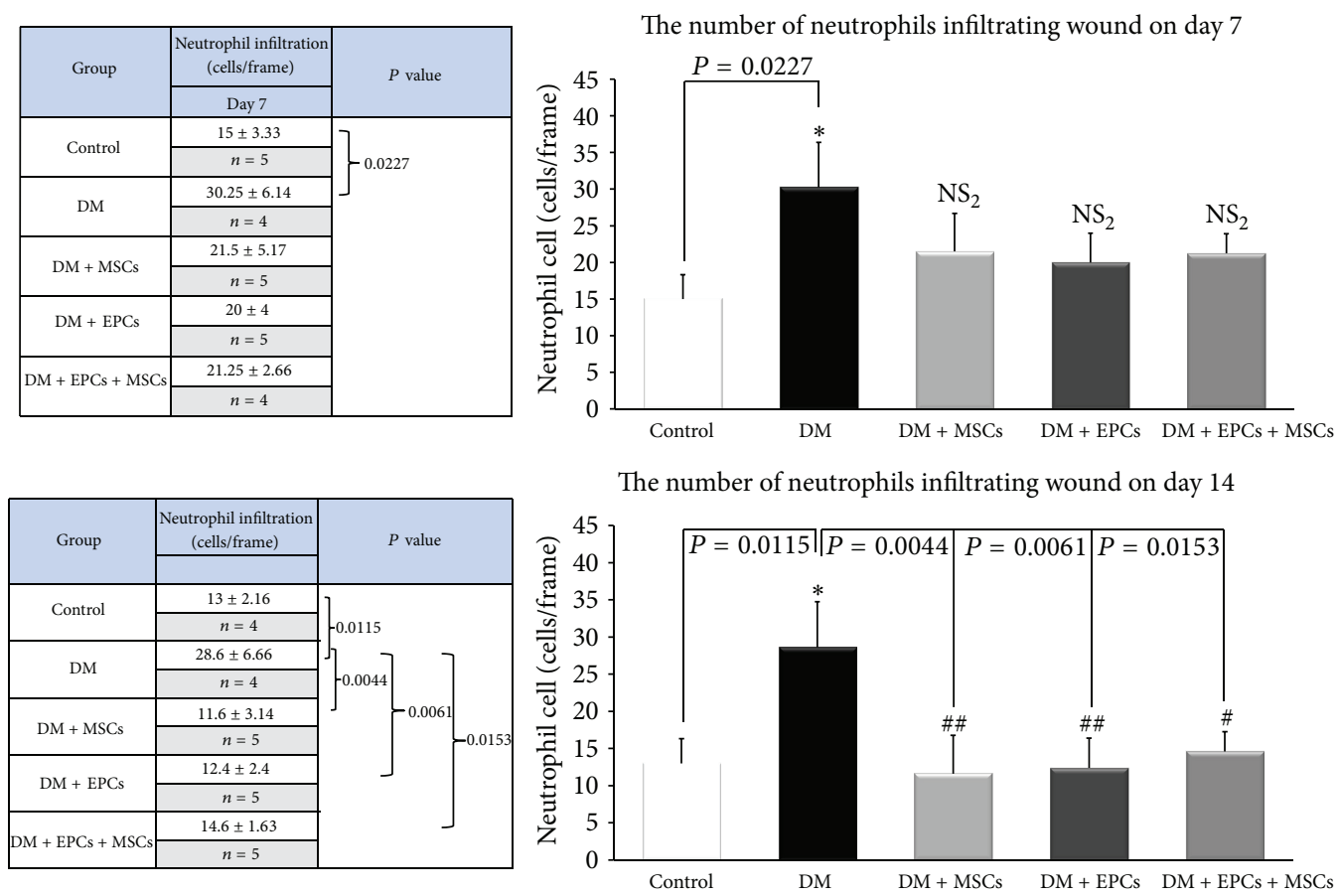

FIGURE 4: The number of infiltrating neutrophils in the control group with implanted fibrin gel (control), diabetic wound group with implanted fibrin gel (DM), diabetic wound group with implanted fibrin gel and MSCs (DM + MSCs), diabetic wound group with implanted fibrin gel and EPCs (DM + EPCs), and diabetic wound group with implanted fibrin gel and combined MSCs and EPCs (DM + MSCs + EPCs) on day 7 and day 14 is shown. The data are presented as the means \pm SEM. ${ }^{*} P<0.05$ : significant difference compared to the control group. ${ }^{\#} P<$ 0.05: significant difference compared to the DM group. ${ }^{\# \#} P<0.01$ : significant difference compared to the diabetic group. $\mathrm{NS}_{2}$ : no significant difference compared to the DM group.

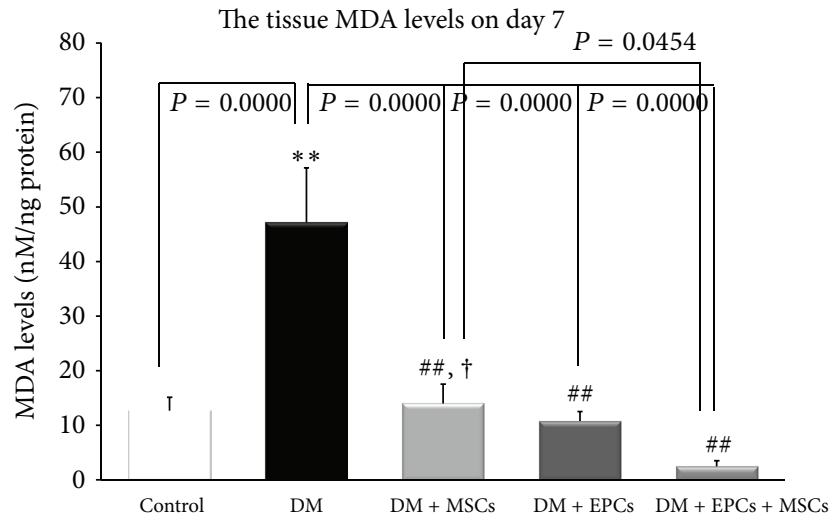

(a)

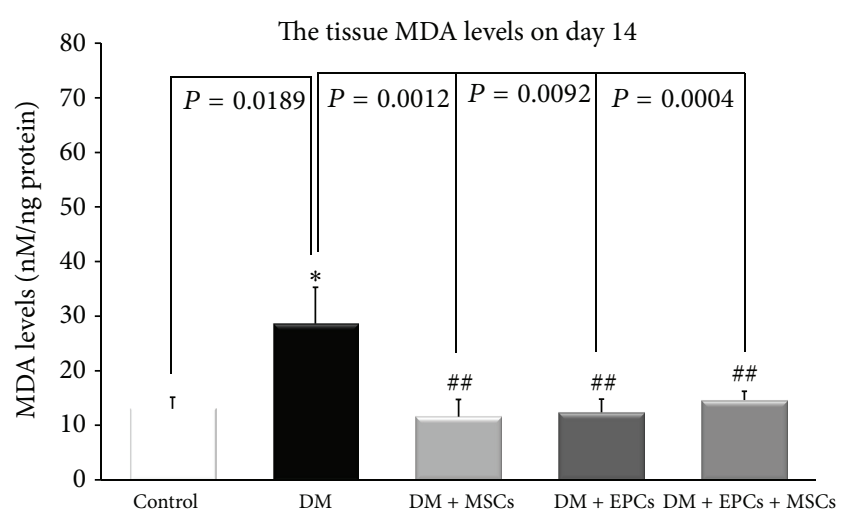

(b)

FIGURE 5: The tissue malondialdehyde levels (MDA) in the control group with implanted fibrin gel (control), diabetic wound group with implanted fibrin gel (DM), diabetic wound group with implanted fibrin gel and MSCs (DM + MSCs), diabetic wound group with implanted fibrin gel and EPCs (DM + EPCs) and diabetic wound group with implanted fibrin gel and combined MSCs and EPCs (DM + MSCs + EPCs) on day 7 and day 14 are shown. ${ }^{*} P<0.05$ : significant difference compared to the control group. ${ }^{* *} P<0.0001$ : significant difference compared to the control group. ${ }^{\# \#} P<0.01$ : significant difference compared to the DM group. ${ }^{\dagger} P<0.05$ : significant difference compared to the DM + MSCs + EPCs group.

the DM group. However, no significant difference between all of the groups on day 14 was observed. According to the normal process of wound healing, increased VEGF is normally increased maximally on day 7 and then declines at day 14 after the emergence of neocapillaries [16].
Taken together, these results also suggested that combined EPCs and MSCs have the potential to increase VEGF similar to the DM + EPCs and DM + MSCs groups. There was also no significant difference among the treatment groups on day 7. 


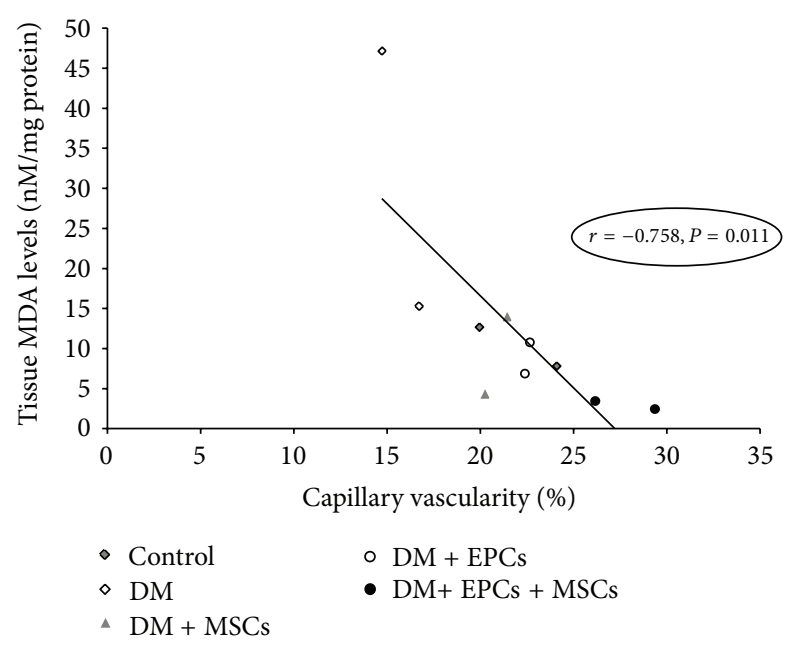

FIGURE 6: Relationship between capillary vascularity (\%CV) and tissuemalondialdehyde levels (MDA). Data were obtained from the mean of each group on day 7 and day 14 .

It is thought that MSCs can increase chemoattractive and mitogenic factors, including VEGF, similar to EPCs [6-8]. Moreover, it has been reported that MSCs may also increase the number of cells positive for CD34, C-kit, or Flk-1, which are endothelial lineage cell markers and increase the recruitment of endothelial cells and endothelial progenitor cells into the wound [17-21]. As shown in our study (Figure 2), the combined DM + EPCs + MSCs group significantly demonstrated the highest increase in capillary vascularity on both day 7 and day 14 . This result may be the first in vivo evidence indicating the synergistic effect of these two cell types on enhancing angiogenesis in the diabetic wound.

The present study also demonstrated that, on day 14, the number of infiltrating neutrophils in the DM + MSCs, $\mathrm{DM}+\mathrm{EPCs}$, and DM + EPCs + MSCs groups was decreased compared to the DM group (Figure 4).

Taken together, the results of the present study indicated that the combined treatment of EPCs and MSCs could reduce neutrophil infiltration, improve angiogenesis, and increase VEGF levels, which could promote a better outcome of wound closure in a diabetic mice model.

These results indicated that tissue MDA levels in the DM group were significantly increased compared to the control group. Combined EPC and MSC treatment significantly demonstrated the best potential in reducing the extent of wound-induced oxidative stress on day 7. The increase in ROS is a common characteristic at the wound site. In a previous study performed using an excision wound mouse model, the major product of lipid peroxidation, 4-hydroxy-2-nonenal (4-HNE), was reported [22]. Interestingly, coimmunostaining revealed that 4-HNE mainly colocalized with neutrophils; thus, it was suggested that the respiratory burst of these inflammatory cells results in the production of superoxide, which in turn causes lipid peroxidation.

Interestingly, low levels of ROS are required for the defense against invading pathogens [23], as well as for mediating intracellular signaling [24]. However, excessive amounts of ROS are deleterious due to their high reactivity.

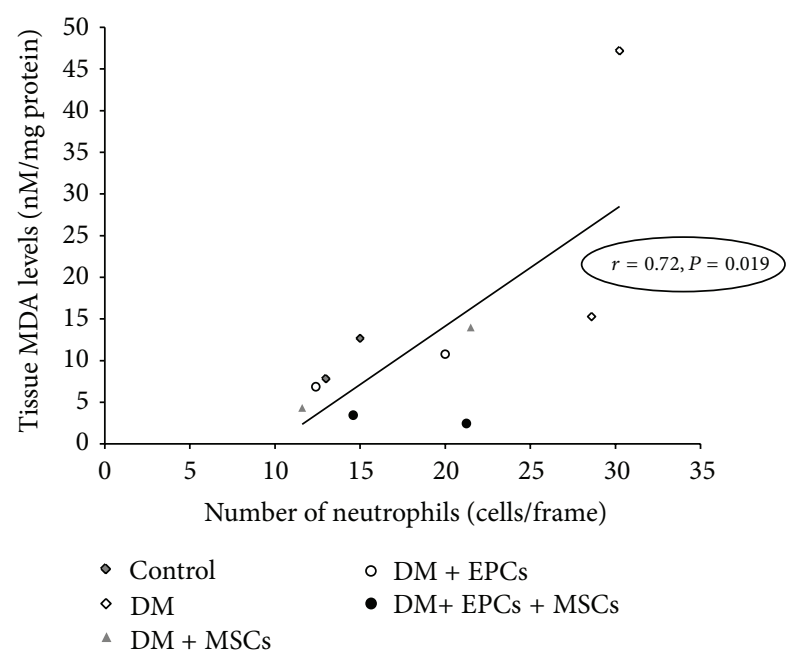

FIGURE 7: Relationship between number of neutrophils (Cells/ frame) and tissuemalondialdehyde levels (MDA). Data were obtained from the mean of each group on day 7 and day 14 .

These results suggested that combined EPC and MSC treatment could improve the wound healing process due to their consequential effects on angiogenesis and antiinflammation, resulting in a decrease in antioxidative stress. We proposed that combined EPC and MSC treatment could inhibit diabetes-induced oxidative stress in the wound area via two potential mechanisms. The first mechanism involves the promotion of sufficient blood perfusion to enhance angiogenesis in the wound area by combined treatment with EPCs and MSCs. Thus, hypoxia cannot amplify the early inflammatory response, thereby inhibiting prolonged injury. The second mechanism is that combined EPC and MSC treatment inhibits oxidative stress produced from the inflammatory cells, thereby restoring the balance between ROS and the antioxidant capacity within the wound area.

Correlation analyses between tissue MDA levels and $\% \mathrm{CV}$ and between MDA levels and the extent of neutrophil infiltration strongly support these two potential mechanisms (Figures 6 and 7). This correlation indicated that, in the treatment groups, the \%CV increased with the reduced number of infiltrating neutrophils and significantly corresponded to the changes in wound ROS content, which is reflected at the MDA level.

Taken together, we showed that combined EPC and MSC administration significantly improved vascular function in diabetic wound mice. These data may have important implications for the potential use of MSCs as a cellular therapy and reduce the use of EPCs in diabetic wound conditions characterized by compromised vascularity and wound closure. Our study demonstrated that combined EPC and MSC treatment might provide benefits for diabetic patients and lower the risk of organ loss.

\section{Abbreviations}

bFGF: Basic fibroblast growth factor

DM: Diabetes mellitus 
DMEM: Dulbecco's Modified Eagle's Medium

EBM-2: Endothelial cell basal medium 2

EPCs: Endothelial progenitor cells

FBS: Fetal bovine serum

MDA: Malondialdehyde levels

MSCs: Mesenchymal stem cells

PBS: $\quad$ Phosphate-buffered saline

PDGF: Platelet-derived growth factor

ROI: $\quad$ Region of interest

ROS: Reactive oxygen species

SDF-1: Stromal-derived factor-1

STZ: $\quad$ Streptozotocin

TGF- $\beta$ : Transforming growth factor- $\beta$

VEGF: Vascular endothelial growth factor

\%WC: Percentage of wound closure

\%CV: Percentage of capillary vascularity

4-HNE: 4-Hydroxy-2-nonenal.

\section{Acknowledgments}

This study was supported by the Ratchadapiseksompotch Fund of the Faculty of Medicine, the 90th Anniversary Research Fund, the Graduate School at Chulalongkorn University, Thailand, and the Higher Education Research Promotion and National Research University Project of Thailand, Office of the Higher Education Commission (HR1166I). The authors have no conflict of interests to report.

\section{References}

[1] M. Reimann, E. Bonifacio, M. Solimena et al., "An update on preventive and regenerative therapies in diabetes mellitus," Pharmacology and Therapeutics, vol. 121, no. 3, pp. 317-331, 2009.

[2] R. E. Pecoraro, G. E. Reiber, and E. M. Burgess, "Pathways to diabetic limb amputation. Basis for prevention," Diabetes Care, vol. 13, no. 5, pp. 513-521, 1990.

[3] G. E. Reiber, R. E. Pecoraro, and T. D. Koepsell, "Risk factors for amputation in patients with diabetes mellitus. A case-control study," Annals of Internal Medicine, vol. 117, no. 2, pp. 97-105, 1992.

[4] G. K. Kolluru, S. C. Bir, and C. G. Kevil, "Endothelial dysfunction and diabetes: effects on angiogenesis, vascular remodeling, and wound healing," International Journal of Vascular Medicine, vol. 2012, Article ID 918267, 30 pages, 2012.

[5] R. Blakytny and E. Jude, "The molecular biology of chronic wounds and delayed healing in diabetes," Diabetic Medicine, vol. 23, no. 6, pp. 594-608, 2006.

[6] L. Debin, J. Youzhao, L. Ziwen, L. Xiaoyan, Z. Zhonghui, and C. Bing, "Autologous transplantation of bone marrow mesenchymal stem cells on diabetic patients with lower limb ischemia," Journal of Medical Colleges of PLA, vol. 23, no. 2, pp. 106-115, 2008.

[7] T. Iwase, N. Nagaya, T. Fujii et al., "Comparison of angiogenic potency between mesenchymal stem cells and mononuclear cells in a rat model of hindlimb ischemia," Cardiovascular Research, vol. 66, no. 3, pp. 543-551, 2005.

[8] Y. Wu, L. Chen, P. G. Scott, and E. E. Tredget, "Mesenchymal stem cells enhance wound healing through differentiation and angiogenesis," Stem Cells, vol. 25, no. 10, pp. 2648-2659, 2007.
[9] B. Zhou, X. Cao, Z. Fang et al., "Prevention of diabetic microangiopathy by prophylactic transplant of mobilized peripheral blood mononuclear cells," Acta Pharmacologica Sinica, vol. 28, no. 1, pp. 89-97, 2007.

[10] V. Falanga, S. Iwamoto, M. Chartier et al., "Autologous bone marrow-derived cultured mesenchymal stem cells delivered in a fibrin spray accelerate healing in murine and human cutaneous wounds," Tissue Engineering, vol. 13, no. 6, pp. 1299-1312, 2007.

[11] M. D. Menger and H. Lehr, "Scope and perspectives of intravital microscopy-bridge over from in vitro to in vivo," Immunology Today, vol. 14, no. 11, pp. 519-522, 1993.

[12] J. Somchaichana, T. Bunaprasert, and S. Patumraj, "Acanthus ebracteatus Vahl. ethanol extract enhancement of the efficacy of the collagen scaffold in wound closure: a study in a fullthickness-wound mouse model," Journal of Biomedicine and Biotechnology, vol. 2012, Article ID 754527, 8 pages, 2012.

[13] M. Masutani, H. Suzuki, N. Kamada et al., "Poly(ADPribose) polymerase gene disruption conferred mice resistant to streptozotocin-induced diabetes," Proceedings of the National Academy of Sciences of the United States of America, vol. 96, no. 5, pp. 2301-2304, 1999.

[14] S. Lenzen, "The mechanisms of alloxan- and streptozotocininduced diabetes," Diabetologia, vol. 51, no. 2, pp. 216-226, 2008.

[15] A. Medina, P. G. Scott, A. Ghahary, and E. E. Tredget, "Pathophysiology of chronic nonhealing wounds," Journal of Burn Care and Rehabilitation, vol. 26, no. 4, pp. 306-319, 2005.

[16] J. Li, J. Chen, and R. Kirsner, "Pathophysiology of acute wound healing," Clinics in Dermatology, vol. 25, no. 1, pp. 9-18, 2007.

[17] D. R. Knighton, G. D. Phillips, and V. D. Fiegel, "Wound healing angiogenesis: indirect stimulation by basic fibroblast growth factor," Journal of Trauma, vol. 30, no. 12, supplement, pp. S134S144, 1990.

[18] S. S. Brem, P. M. Gullino, and D. Medina, "Angiogenesis: a marker for neoplastic transformation of mammary papillary hyperplasia," Science, vol. 195, no. 4281, pp. 880-882, 1977.

[19] L. E. Huang, Z. Arany, D. M. Livingston, and H. Franklin Bunn, "Activation of hypoxia-inducible transcription factor depends primarily upon redox-sensitive stabilization of its $\alpha$ subunit," Journal of Biological Chemistry, vol. 271, no. 50, pp. 32253-32259, 1996.

[20] S. Enoch and D. J. Leaper, "Basic science of wound healing," Surgery, vol. 26, no. 2, pp. 31-37, 2008.

[21] A. J. Guidi, S. J. Schnitt, L. Fischer et al., "Vascular permeability factor (vascular endothelial growth factor) expression and angiogenesis in patients with ductal carcinoma in situ of the breast," Cancer, vol. 80, no. 10, pp. 1945-1953, 1997.

[22] N. Ojha, S. Roy, G. He et al., "Assessment of wound-site redox environment and the significance of Rac2 in cutaneous healing," Free Radical Biology and Medicine, vol. 44, no. 4, pp. 682-691, 2008.

[23] B. D’Autréaux and M. B. Toledano, "ROS as signalling molecules: mechanisms that generate specificity in ROS homeostasis," Nature Reviews Molecular Cell Biology, vol. 8, no. 10, pp. 813-824, 2007.

[24] A. E. Sakallıoğlu, Ö. Başaran, B. H. Özdemir et al., "Local and systemic interactions related to serum transforming growth factor- $\beta$ levels in burn wounds of various depths," Burns, vol. 32, no. 8, pp. 980-985, 2006. 

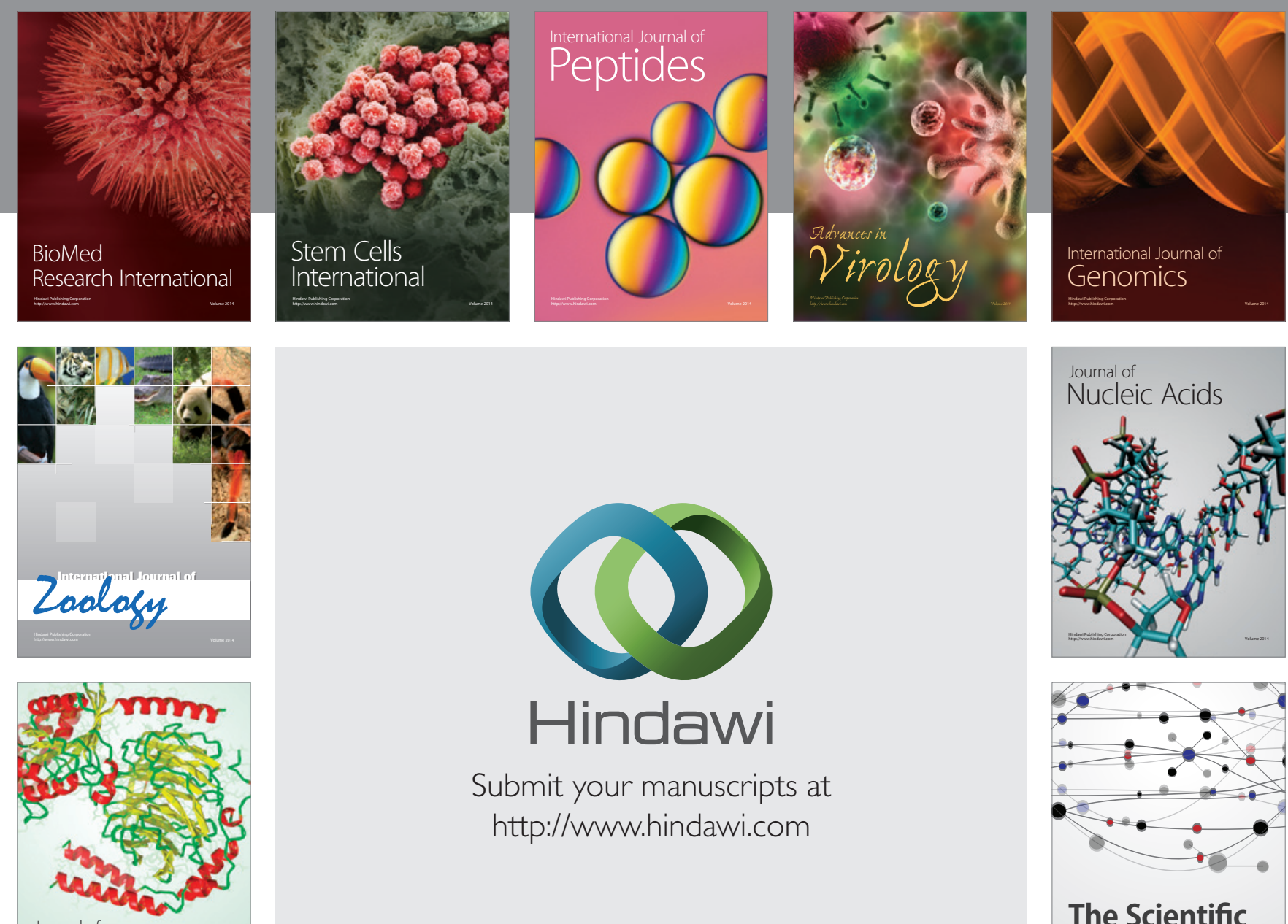

Submit your manuscripts at

http://www.hindawi.com

Journal of
Signal Transduction
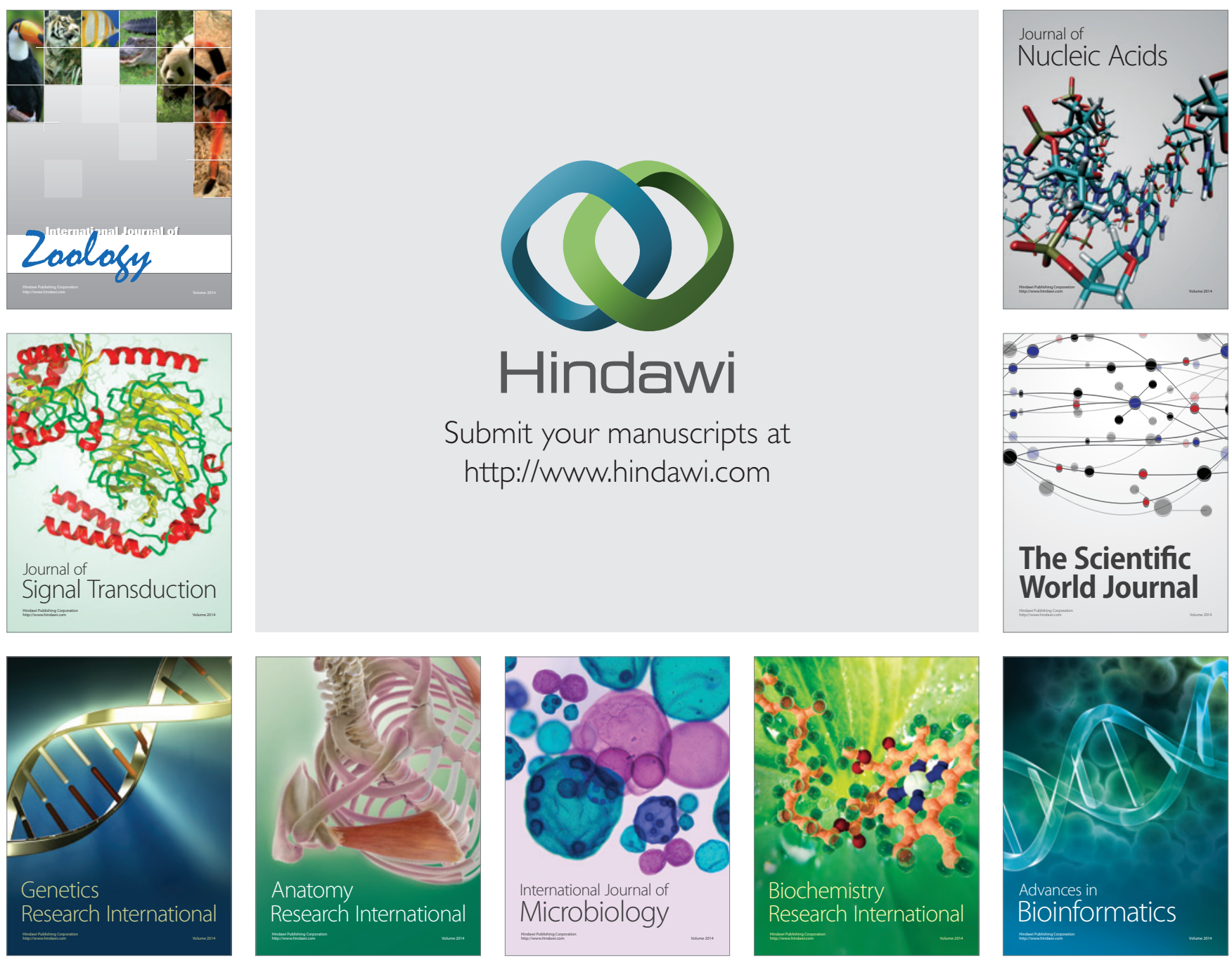

The Scientific World Journal
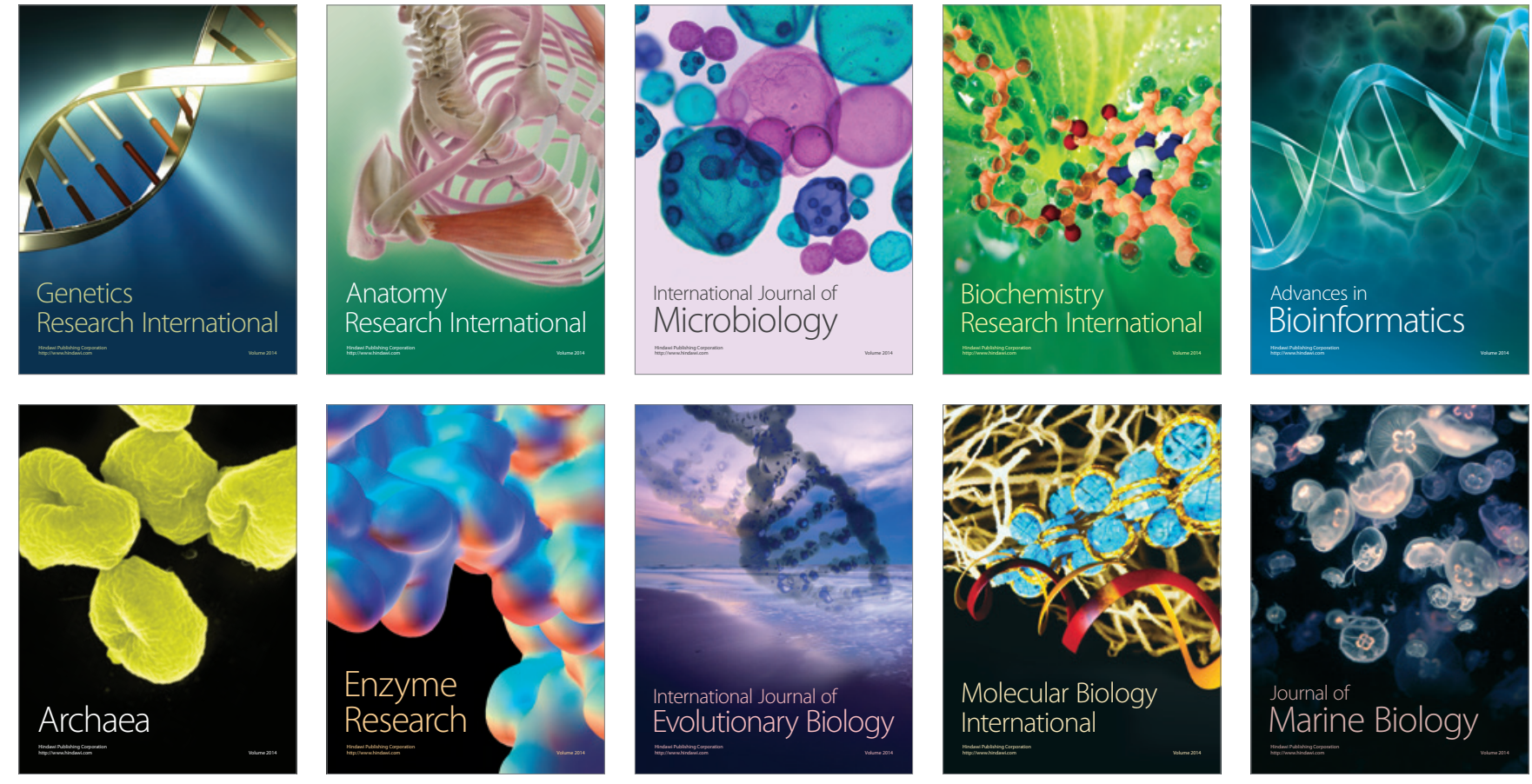